\title{
Palliative Treatment and the Role of Surgical Resection in Gastric Cancer
}

\author{
Martin S. Karpeh Jr. \\ Department of Surgery, Beth Israel Medical Center, and Division of Surgical Oncology, \\ Continuum Cancer Centers of New York, New York, N.Y., USA
}

\section{Key Words}

Gastric adenocarcinoma · Noncurative resection · Palliation

\begin{abstract}
Adenocarcinoma of the stomach is often diagnosed in the late stages of the disease. Surgical resection of all gross and microscopic disease is essential for curative treatment. Complete resection is often not achievable when patients present with advanced stage IV cancer. In the absence of symptoms, chemotherapy without resection has been the standard of care in most major centers. With improvements in response to chemotherapy and less invasive surgical approaches, patients with metastatic gastric cancer have had better survival outcomes than in the past. The challenge today when treating these patients is in defining who will benefit from more aggressive interventions. Reviewing the literature for guidance is difficult because the goals of treatment are often not clearly defined. Finding the proper balance of aggressiveness needed to extend survival while preserving and maximizing quality of life is a decision that clinicians have to make with increasing frequency. This review will attempt to provide a framework to aid in determining what role, if any, gastrectomy has in the management of patients with stage IV gastric cancer.
\end{abstract}

Copyright $\odot 2013$ S. Karger AG, Basel
(C) 2013 S. Karger AG, Basel

0253-4886/13/0302-0174\$38.00/0

\section{Introduction}

Gastric adenocarcinoma is the second leading cause of cancer deaths globally. Despite improvements in overall survival, the majority of patients diagnosed with this disease worldwide present with metastatic disease. The goals of treatment are often noncurative and directed towards symptom relief. Chemotherapy and other targeted systemic options are the major components of treatment in stage IV disease. Improvements in the response to systemic therapies have brought the role of resecting the primary tumor in stage IV gastric cancer into question. Gastrectomy and metastasectomy have traditionally played a purely palliative role, relieving tumor-related symptoms. Assessing the potential role of surgery in these patients is difficult because the data is retrospective. The original intent of treatment in these studies is usually not defined. Palliation, which should refer specifically to the relief of symptoms, has unfortunately been widely applied to describe all noncurative treatments. When examining the role of gastrectomy in the palliative treatment of gastric cancer, the outcome measured is limited to survival. The true benefit or cost of resection versus a less invasive approach or chemotherapy alone cannot be adequately examined by taking such a limited view. It is important to clearly define the goal of therapy since the intent can

\section{KARGER}

E-Mail karger@karger.com

www.karger.com/dsu
Martin S. Karpeh Jr., M.D.

Department of Surgery, 16th Floor Baird Hall, Beth Israel Medical Center 350 East 17 th Street

New York, NY 10003 (USA)

E-Mail mkarpeh@chpnet.org 
range from providing effective relief of gastric outlet obstruction to extending patient survival in the absence of tumor-related symptoms. In order to understand the role of surgery in advanced metastatic gastric cancer, defining the goals of palliation are essential [1]. While survival is always an important endpoint, preserving quality of life (QoL), in many cases, may be the most appropriate and potentially achievable goal. To date, the majority of surgical papers on this subject have focused predominantly on survival as the measure of palliative success. The quality of any perceived survival benefit in these studies is either not addressed or minimized. Properly designed prospective studies to guide decision-making regarding the role of surgery in stage IV gastric cancer are lacking. A number of large retrospective studies have suggested that operative intervention improves the survival of these patients. Factors such as the extent and distribution of the disease, patient performance status, extent of the resection, and the response to chemotherapy are addressed in this review to assess the role of surgical intervention in the treatment of stage IV gastric cancer.

\section{Defining Palliative Intent}

The varied use of the term palliation in the surgical literature has complicated our ability to understand the role of surgery in the management of patients with stage IV gastric cancer. To palliate refers to the reduction, easing, or moderation of the intensity or virulence of a disease, and yet palliation in most surgical studies is usually applied to any noncurative treatment. The assessment of a 'palliative gastrectomy' is often made solely on the basis of overall survival, but relief of pain, improvement in performance status, and other measures of a health-related QoL are lacking. Surgical palliation should be consistently defined as a surgical intervention that is primarily intended to relieve symptoms or improve QoL [2]. It is not possible to palliate in the absence of symptoms. When assessing retrospective data, it is critical to identify the intent of the treatment.

In an attempt to better understand the impact of defining the intent of treatment in patients undergoing noncurative surgery, we compared the survival outcome of asymptomatic patients undergoing noncurative gastrectomy from those undergoing truly palliative symptomrelieving gastric resections. A retrospective analysis of 307 stage IV gastric cancer patients was performed [3]. All operations were reviewed and defined as either truly palliative, meaning that the procedure was performed for

Palliative Treatment in Gastric Cancer relief of symptoms, pain control, or improvement in QoL. The operation was palliative in $48 \%$ (147/307) and nonpalliative in $52 \%$. The overall operative mortality was $6 \%$ and morbidity was $54 \%$ with no significant differences between the two groups. The overall median survival for all resections was 10.6 months. Survival for those undergoing surgery for symptom relief was only 8.3 months compared to 13.5 months for the nonpalliative group $(\mathrm{p}<0.001)$. Palliative operations included an esophageal anastomosis less frequently (46 vs. $69 \%, \mathrm{p}<0.001)$ and had a less extensive lymphadenectomy performed compared with nonpalliative operations. The degree of symptoms tended to correlate with disease burden. The results illustrate that the intent of noncurative surgery has a direct impact on survival and must be stated for a meaningful interpretation of results. Unfortunately, the degree and duration of palliation in the palliative operation group was not retrievable from the records. In studies of stage IV gastric cancer patients undergoing noncurative operations, measures of the degree and duration of the palliation need to be included. Palliation has its own set of indications and goals, and should be evaluated as its own distinct endpoint.

\section{Noncurative Resections}

Resection of all gross and microscopic disease is the established goal of a curative operation. Procedures that fall short of achieving that goal should be referred to as noncurative rather than the common misnomer palliative. Noncurative resections may or may not be palliative. Improvements in surgical care and outcomes have brought into question the role of resecting the primary in stage IV gastric cancer. Mahar et al. [4] performed a systematic review of English language studies published from 1985 to 2009 of surgery for noncurative gastric cancer. Fifty-nine articles reporting 30 or more patients were included. The data was predominantly retrospective and from single institutions. Long-term survival was the principle outcome measured. The median survival following noncurative gastrectomy ranged from 3 to 24 months. The wide range in median survival highlights the wide variability in these data. The lack of clear selection criteria, variability in tumor burden, and performance status present major challenges when interpreting results.

A recent study not included in the above review analyzed data from the United States Surveillance, Epidemiology and End Results (SEER) Program database. The authors examined the relationship between gastrectomy 
Table 1. Type of operation performed on stage IV gastric cancer patients undergoing cancer-directed gastric surgery from the SEER database $(\mathrm{n}=8,488)$ : January 1,1988 through December 31, 2005

\begin{tabular}{lc}
\hline Procedure type & $\mathrm{n}(\%)$ \\
\hline Local tumor destruction & $243(2.9)$ \\
Partial gastrectomy & $4,358(51.3)$ \\
Total/near total gastrectomy & $1,430(16.8)$ \\
Gastrectomy with en bloc resection & $1,919(22.6)$ \\
Gastrectomy not otherwise specified & $126(1.5)$ \\
Surgery not otherwise specified & $389(4.6)$ \\
Other cancer-directed surgery & $23(0.3)$ \\
\hline
\end{tabular}

and survival in stage IV gastric cancer patients treated between 1988 and 2005. An attempt was made to minimize selection bias by dividing patients into three groups depending on whether cancer-directed surgery was recommended and performed. The three groups comprised patients who were: (1) recommended for and received cancer-directed surgery, (2) recommended for cancer-directed surgery but did not have surgery or underwent noncancer-directed surgery, and (3) not recommended for cancer-directed surgery and no surgery was performed or the surgery was noncancer-directed surgery. Of the 66,751 patients identified with gastric cancer, 23,830 had stage IV disease and had their outcomes analyzed. Cancer-directed surgery was recommended for 10,910 of the patients (45.7\%). No information was available in the SEER registry regarding the symptom profile of the patients or their performance status at the time of surgery. Among the group who were recommended to have cancer-directed surgery, 8,488 (77.8\%) actually had an operation. The types of operations are shown in table 1 . On multivariate analysis, the probability of receiving cancer-directed surgery once it was recommended decreased significantly for patients who were 50 years of age or older, male, had a marital status other than married, and had M1 disease as opposed to M0. Predictors associated with receiving cancer-directed surgery were 'other' race (which included Asian) and tumor grades III and IV. Overall median survival for all stage IV patients was only 4 months. Patients who were recommended to have and received cancer-directed surgery had a significant survival advantage over those who did not have surgery regardless of the recommendation. All types of cancer-directed surgery conferred some survival advantage over no surgery. The authors found that patients who were not recommended for cancer-directed surgery as well as patients who were recommended but did not receive cancer-directed surgery had a nearly twofold increased risk of death compared to patients who were recommended and received cancer-directed surgery. In this cohort, male gender, age older than 50 years, black race, tumor grade higher than I, and having surgery in the years prior to 2003-2005 all had a significant increased risk of death. From this data, African-American males over the age of 50 carry the greatest risk of not benefiting from cancer-directed surgery. These data need to be interpreted cautiously given the lack of information regarding social and economic factors, tumor factors, or other treatments. We have no data indicating why certain patients were recommended for a cancer-directed operation and others were not. There was also no data regarding the use or response to chemotherapy. Clearly, the surgeons were able to select those patients they felt were healthy enough to undergo an operation.

The volume and distribution of metastatic disease is an important factor when evaluating patients with stage IV gastric cancer. In a study of 253 gastric cancer patients presenting with synchronous distant metastasis to the Department of Surgery in Ruijin Hospital, China, the 5 -year survival rate was $6.5 \%$ for patients in the resection group and $0 \%$ for patients in the nonresection group $(\mathrm{p}<$ 0.001 ) [5]. A multivariate analysis revealed that patients with liver metastasis, peritoneal dissemination, or those who were not resected had the worse prognosis, and the survival difference between the resection and nonresection groups was only observed in patients with a single site of peritoneal dissemination $(\mathrm{p}<0.001)$. In patients with single liver metastasis, distant nodal involvement or multiple metastatic sites, there was no survival benefit from noncurative gastrectomy. Patient selection is critical to determining if there is a role for resection in stage IV disease. The role of chemotherapy was not addressed in this study.

An analysis of data from the Dutch randomized control trial of D1 versus D2 lymphadenectomy identified 285 patients who had noncurative resections because of either residual liver, peritoneal metastases, distant lymph node metastases, or remnant tumor. The number of noncurability signs quantified tumor load retrospectively. Within this cohort of incurable patients, the resected patients had a significantly longer median survival than patients treated without resection. This survival advantage disappeared when two or more positive signs of noncurability were present [6]. Age less than 70 years was found to be associated with less morbidity and mortality and 
Table 2. Prognostic factors of outcome following noncurative gastrectomy for gastric cancer

\begin{tabular}{lll}
\hline Prognostic factor & HR & 95\% CI \\
\hline Single agent vs. combination chemo & 1.35 & $1.068-1.721$ \\
Previous palliative gastrectomy vs. none & 12 & $7.969-18.099$ \\
Histological grade III vs. grade I, II & 1.39 & $1.098-1.782$ \\
Hepatic metastasis vs. none & 1.6 & $1.246-2.073$ \\
Abdominal metastasis vs. none & 1.33 & $1.039-1.715$ \\
CA 72-4 $>7$ U/l & 1.39 & $1.026-1.887$ \\
LDH $>225$ U/l & 1.72 & $1.336-2.236$ \\
Blood transfusions vs. none & 1.58 & $1.213-2.082$ \\
Weight loss $>5 \%$ & 1.96 & $1.352-2.853$ \\
\hline
\end{tabular}

Low risk $=0-2$ factors; intermediate risk $=3-6$ factors; high risk $=7-9$ factors.

better survival following resection [7]. Patients with the fewest number of noncurability signs were significantly more likely to undergo resection which underscores the selection bias when evaluating the role of gastrectomy in the noncurative setting. No specific data were given concerning the surgeons' selection criteria.

In another study of 311 patients who had noncurative gastrectomy for gastric cancer, seventeen clinicopathological and therapeutic parameters were retrospectively evaluated for their influence on overall survival [8] and nine factors were found in a multivariate analysis to independently influence survival (table 2). Patients were risk stratified by the number of factors into low ( $0-2$ factors), intermediate (3-6 factors), or high (7-9 factors). The median survival was 76,40 , and 11 weeks, respectively. Noncurative gastrectomy when combined with chemotherapy was associated with improved survival.

These studies collectively suggest that improved survival after resection is also associated with lower tumor burden. It can be safely presumed that surgeons' selection criteria include performance status, which is likely to be better than those patients not selected for resection.

\section{Gastrectomy as a Component of Multimodality Therapy}

The latest Cochrane review found that the use of chemotherapy improved survival over best supportive care in the management of patients with incurable gastric cancer. The evidence also favored combination chemotherapy over single-agent approaches [9]. Patients with meta- static gastric cancer have traditionally not been considered operative candidates and are offered systemic therapy as a monotherapy. Palliative resection of the primary tumor has been considered irrelevant to the outcome and has been recommended only for palliation of symptoms. However, improvements in response to combination chemotherapy [10] and in surgical management have raised the question of whether gastrectomy following induction chemotherapy has a role in the management of stage IV gastric cancer. Twelve centers from Japan reported results on 28 stage IV gastric cancer patients who underwent surgery with curative intent following treatment with S-1-based chemotherapy [11]. Patients who were enrolled had clinical stage T4 tumors (9 patients), para-aortic node metastasis (15 patients), peritoneal metastasis ( 7 patients), and liver metastasis (4 patients). The study reported that $93 \%$ of the patients achieved an R0 resection. Four patients had a complete pathological response. The median survival was 29 months with $34.4 \%$ surviving 5 years. On multivariate analysis, histological tumor length of $5.0 \mathrm{~cm}$ or larger was the only significant prognostic factor. Pathologic tumor size may be a surrogate for tumor burden, but it may also represent tumor response. The authors concluded that gastrectomy following S-1-based chemotherapy for stage IV gastric cancer was a safe and effective treatment. Others have also suggested that survival time and the duration of palliation were significantly longer in selected patients treated with the combination of chemotherapy followed by gastrectomy in contrast to treatments without resection [12]. A retrospective review of 36 patients with metastatic disease undergoing resection with therapeutic intent following chemotherapy found that those patients with persistent detectable metastatic disease did poorly [13]. The results raise further questions regarding which stage IV patients, who are relatively symptom free, benefit from resection.

\section{Quality of Life}

Surgery has traditionally played a significant role in relieving symptoms from advanced gastric cancer [14], but in most of the surgical literature, the duration and degree of palliation are not directly measured. Better than expected median survivals have been documented in selected patients with stage IV gastric cancer following resection, but virtually all of these patients ultimately progress and succumb to their disease. Therefore, an accurate assessment of health-related QoL in patients with 
metastatic gastric cancer is essential to inform clinical decision-making. The growth of endoscopic and other minimally invasive procedures has broadened the options for symptom control, further emphasizing the importance of understanding which intervention is best for a given clinical setting. QoL questionnaires that have been validated to specifically measure the QoL of patients with gastric cancer are available [15], yet few reports on palliative gastrectomy use these tools [16]. Most retrospective papers have relied on hospital-free survival as a surrogate for QoL. The group from the Miyagi Cancer Center Hospital in Japan studied 95 patients undergoing palliative operations for gastric cancer [17] and found that overall survival and hospital-free survival for 3 months or longer was higher following palliative gastrectomy than for gastrojejunostomy. Keeping patients out of the hospital is a reasonable quality goal; however, these findings again demonstrate clear selection bias in favor of those being resected. When accounting for the extent of disease, the subset of patients who had a total gastrectomy in the presence of $\mathrm{P} 2$ or $\mathrm{P} 3$ peritoneal disease showed a poorer outcome for survival and more frequent hospitalizations than total gastrectomy performed with P0 or P1 and distal gastrectomy. Gastrojejunostomy and total gastrectomy performed with $\mathrm{P} 2$ or $\mathrm{P} 3$ peritoneal dissemination had no beneficial effect on survival or the number of hospitalizations. Hospital-free survival was also studied in retrospective analysis of noncurative resection from Memorial Sloan Kettering Cancer Center [18]. Time spent without symptoms requiring additional procedures or hospitalization was increased in patients who had multiple sites of metastases and/or had suffered a major procedure-related complication. Unfortunately, there are no surgical data using established validated QoL instruments to assess the impact of gastrectomy in the noncurative setting. The data again demonstrate that multiple factors influence the survival of these patients. Proper assessment of noncurative gastrectomy requires prospective consideration of tumor burden, patient physical status, and treatment factors to make informative comparisons. Prospectively designed studies using disease-specific validated QoL measures will better assist the treatment decisionmaking process [16].

\section{Prophylactic Gastrectomy}

The concept of removing a primary gastric malignancy in the face of distant metastasis to avoid future symptoms of obstruction or bleeding has not been supported by the literature. One hundred and sixty-five patients with metastatic gastric cancer diagnosed at laparoscopy and treated with chemotherapy only were studied to investigate the need for intervention for symptom control [19]. The distribution of metastases was as follows: $74 \%$ had peritoneal only $(9 \%=\mathrm{P} 1 ; 35 \%=\mathrm{P} 2 ; 30 \%=\mathrm{P} 3), 10 \%$ had liver only, and $16 \%$ had both liver and peritoneal disease. These patients were healthy enough for surgery with an ECOG functional performance status of $0-1$ in $84 \%$ or 2 in $16 \%$. At a median follow-up time of 4 months following laparoscopy (1-35 months), half of the patients underwent some form of intervention. Laparotomy was necessary in only $12 \%$ of the patients. The majority of these patients were managed with endoscopic and radiologic procedures or with radiation therapy. Interventions were needed for gastric obstruction in 33\%, tumor bleeding in $8 \%$, and perforation in $1 \% ; 20 \%$ of the time the intervention was for a metastasis-related complication. One patient died due to intervention-related complications. More than one intervention (maximum: 4) was performed in $21 \%$ of patients. Median survival for the group was 10 months, with $39 \%$ surviving 1 year. As expected, survival correlated significantly with better functional performance status and with having limited peritoneal metastasis. Many surgeons considering gastrectomy with therapeutic intent would likely choose patients with these same favorable characteristics and attribute any survival benefit to the operation. With improvements in endoscopic and minimally invasive surgical techniques, intervening as symptoms develop is an appropriate course in managing these patients. More studies are needed to assess when and how to best utilize the multiple choices currently available for symptom management.

\section{Randomized Trails}

The prolonged survival associated with resecting the primary tumor in metastatic gastric cancer has prompted investigators to prospectively study the role of resection. The multiple prognostic factors that influence survival requires a prospective randomized trial. There are currently two large randomized trials underway attempting to address this question. The first study is a large multicenter trial involving 33 centers from the Japan Clinical Oncology Group (JCO 0705) and 15 centers from the Korea Gastric Cancer Association Study (KGCA01) that have begun accrual [20]. Patients with advanced gastric cancer and one noncurable factor are randomized between gastrectomy plus chemotherapy and chemothera- 
py alone. The primary endpoint is overall survival. Progression-free survival and adverse events are secondary endpoints. The chemotherapy regimen of S-1 and cisplatin is started within 8 weeks after surgery. The study is powered at $80 \%$ with a one-sided alpha of $5 \%$. The planned sample size is 330, 165 cases per arm, with 2 years follow-up after 4 years of accrual. The study assumes that gastrectomy will provide a $10 \%$ improvement in overall survival at 2 years. QoL is not a defined outcome measure. A planned interim analysis is pending.

The second trial of gastrectomy and/or metastasectomy plus systemic therapy versus systemic therapy alone has started at the National Cancer Institute in the United States [21]. The endpoint again is overall survival. This study assumes that gastrectomy and/or metastasectomy will produce an 8 -month increase in overall survival. A total of 136 patients over 6 years are planned to meet the study goals. The number of metastatic sites allowed was not defined, but patients with both pulmonary and peritoneal metastases are excluded, as are those with brain metastasis. This study in contrast to the prior trial includes an analysis of QoL using standardized evaluation tools designed for patients with gastric cancer [22]. QoL will be compared between the two study arms at multiple time points to determine the differences between the two arms as well as a change in QoL from baseline.

\section{Conclusion}

Improvements in chemotherapy have increased the number of patients living with metastatic gastric cancer. Patients and surgeons have been tempted to proceed to gastrectomy, plus or minus metastasectomy, with the intent of improving survival even further. The current retrospective literature does not inform the decision-making process since most resected patients are heavily preselected. Palliation should refer specifically to interventions aimed at the relief of symptoms rather than simply incurability. Carefully designed prospective studies are currently accruing to assess key outcomes such as health-related QoL, specific symptom control, and hospital-free survival. Factors such as the extent and distribution of disease, response to chemotherapy, and performance status of the patient are important determinants of outcome that should be controlled to better understand if surgical resection has a role in altering the survival time of stage IV gastric cancer. Fortunately, there are now two well-designed randomized controlled trials that will provide the level of evidence needed to guide the treatment of this increasing subset of gastric cancer patients.

\section{References}

1 Miner TJ, Jaques DP, Tavaf-Motamen H, Shriver CD: Decision making on surgical palliation based on patient outcome data. Am J Surg 1999;177:150-154.

$\checkmark 2$ Miner TJ, Jaques DP, Shriver CD: A prospective evaluation of patients undergoing surgery for the palliation of an advanced malignancy. Ann Surg Oncol 2002;9:696-703.

-3 Miner TJ, Jaques DP, Karpeh MS, Brennan MF: Defining palliative surgery in patients receiving noncurative resections for gastric cancer. J Am Coll Surg 2004;198:1013-1021.

-4 Mahar AL, Coburn NG, Singh S, Law C, Helyer LK: A systematic review of surgery for non-curative gastric cancer. Gastric Cancer 2012;15(suppl 1):S125-S137.

5 Li C, Yan M, Chen J, et al: Survival benefit of non-curative gastrectomy for gastric cancer patients with synchronous distant metastasis. J Gastrointest Surg 2010;14:282-288.

6 Bonenkamp JJ, Sasako M, Hermans J, van de Velde CJ: Tumor load and surgical palliation in gastric cancer. Hepatogastroenterology 2001;48:1219-1221.
7 Hartgrink HH, Putter H, Klein Kranenbarg E, Bonenkamp JJ, van de Velde CJ: Value of palliative resection in gastric cancer. Br J Surg 2002;89:1438-1443.

8 Sougioultzis S, Syrios J, Xynos ID, et al: Palliative gastrectomy and other factors affecting overall survival in stage IV gastric adenocarcinoma patients receiving chemotherapy: a retrospective analysis. Eur J Surg Oncol 2011; 37:312-318.

-9 Wagner AD, Unverzagt S, Grothe W, et al: Chemotherapy for advanced gastric cancer. Cochrane Database Syst Rev 2010;3: CD004064.

10 Cunningham D, Allum WH, Stenning SP, et al: Perioperative chemotherapy versus surgery alone for resectable gastroesophageal cancer. N Engl J Med 2006;355:11-20.

-11 Kanda T, Yajima K, Kosugi S, Ishikawa T, Ajioka Y, Hatakeyama K: Gastrectomy as a secondary surgery for stage IV gastric cancer patients who underwent S-1-based chemotherapy: a multi-institute retrospective study. Gastric Cancer 2012;15:235-244.
12 Lin SZ, Tong HF, You T, et al: Palliative gastrectomy and chemotherapy for stage IV gastric cancer. J Cancer Res Clin Oncol 2008; 134 : 187-192.

13 Gold JS, Jaques DP, Bentrem DJ, et al: Outcome of patients with known metastatic gastric cancer undergoing resection with therapeutic intent. Ann Surg Oncol 2007;14:365372.

14 Lawrence W Jr, McNeer G: The effectiveness of surgery for palliation of incurable gastric cancer. Cancer 1958;11:28-32.

$\checkmark 15$ Lagergren P, Fayers P, Conroy T, et al: Clinical and psychometric validation of a questionnaire module, the EORTC QLQ-OG25, to assess health-related quality of life in patients with cancer of the oesophagus, the oesophago-gastric junction and the stomach. Eur J Cancer 2007;43:2066-2073.

-16 Mahar AL, Coburn NG, Karanicolas PJ, Viola R, Helyer LK: Effective palliation and quality of life outcomes in studies of surgery for advanced, non-curative gastric cancer: a systematic review. Gastric Cancer 2012;15(suppl 1):S138-S145. 
17 Ouchi K, Sugawara T, Ono H, et al: Therapeutic significance of palliative operations for gastric cancer for survival and quality of life. J Surg Oncol 1998;69:41-44.

18 Miner TJ, Karpeh MS: Gastrectomy for gastric cancer: defining critical elements of patient selection and outcome assessment. Surg Oncol Clin N Am 2004;13:455-466, viii.

$\checkmark 19$ Sarela AI, Miner TJ, Karpeh MS, Coit DG, Jaques DP, Brennan MF: Clinical outcomes with laparoscopic stage M1, unresected gastric adenocarcinoma. Ann Surg 2006;243: 189-195.
20 Fujitani K, Yang HK, Kurokawa Y, et al: Randomized controlled trial comparing gastrectomy plus chemotherapy with chemotherapy alone in advanced gastric cancer with a single non-curable factor: Japan Clinical Oncology Group Study JCOG 0705 and Korea Gastric Cancer Association Study KGCA01. Jpn J Clin Oncol 2008;38:504-506.
21 Kerkar SP, Kemp CD, Duffy A, et al: The GYMSSA trial: a prospective randomized trial comparing gastrectomy, metastasectomy plus systemic therapy versus systemic therapy alone. Trials 2009;10:121.

22 Blazeby JM, Conroy T, Bottomley A, et al: Clinical and psychometric validation of a questionnaire module, the EORTC QLQSTO 22, to assess quality of life in patients with gastric cancer. Eur J Cancer 2004;40: 2260-2268. 